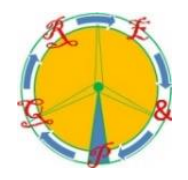

\title{
Advances in Compression of Power Quality Signals
}

\author{
L. C. M. Andrade ${ }^{1}$, T. Nanjundaswamy ${ }^{3}$, M. Oleskovicz ${ }^{1}$, R. A. S. Fernandes ${ }^{2}$ and K. Rose ${ }^{3}$ \\ ${ }^{1}$ Department of Electrical and Computer Engineering, São Carlos School of Engineering, \\ University of São Paulo, São Carlos, SP, Brazil \\ Phone/Fax number:+55 (16) 3373-8142, e-mail: lucianocarli@icmc.usp.br, olesk@sc.usp.br \\ ${ }^{2}$ Department of Electrical Engineering, Federal University of São Carlos, São Carlos, SP, Brazil \\ Phone/Fax number:+55 (16) 3351-9701, e-mail: ricardo.asf@ufscar.br
}

\author{
${ }^{3}$ Department of Electrical and Computer Engineering, University of California, Santa Barbara, CA, USA \\ Phone/Fax number:+1 (805) 893-7024, e-mail: tejaswi@ece.ucsb.edu, rose@ece.ucsb.edu
}

\begin{abstract}
The emerging technology of smart grids relies heavily on monitoring the distribution networks for disturbances using, for example, the devices installed for measuring power quality signals. Obviously, efficient compression of these electrical signals is of paramount importance to allow fast transmission, remote analysis and automation of response to disturbances, as well as archival storage. While prior approaches to compress electrical signal disturbances employed standard effective components such as wavelet transforms, non-uniform quantizers, and entropy coding, the overall system design was largely ad-hoc in the sense that it did not directly account for or adapt to data statistics. Instead, we propose to jointly design all system modules, including transforms, quantizers and entropy coders, within a genetic algorithm-based optimization framework, while accounting for the variation in statistics across different disturbances, within a two-step "classify then compress" procedure. Specifically, we jointly design the family of wavelets to be employed, the non-uniform quantizer structure, and probability tables for the entropy coder, to optimize the ratedistortion trade-off. Experimental results for 8 classes of commonly occurring power quality disturbances, which were synthetically generated to ensure rich and comprehensive training and test sets, validate the effectiveness of the proposed approach with significant performance gains over prior techniques.
\end{abstract}

\section{Keywords: Data Compression, Evolutionary} Computation, Power Quality, Wavelet Transforms.

\section{Introduction}

Signal processing techniques have become indispensable in addressing various challenges encountered in electric power systems (EPS) [1] [2], especially with the emerging technology of smart grids (SG) [3] [4] [5]. These technological advancements require continuous monitoring of the EPS by devices measuring power quality (PQ) signals, which means its effectiveness is critically dependent on efficient compression and transmission of measured signals. However, while various compression approaches for PQ signals have been investigated [6] [7], PQ compression has not reached the maturity of speech, image or video compression [8] [9]. Hence, effective collaboration between researchers in the respective fields of signal compression and EPS is required to provide the compression technology support for proliferation of smart grids, and this paper covers the preliminary work and results for one such foray.

Current approaches for compression of PQ disturbance signals [10] [11] [12] [13] employ tools that are well known to be effective in other coding frameworks. The tools employed include: i) wavelet transforms (WT), to take advantage of its ability to decompose the signals into timefrequency bins of varying resolutions; ii) non-uniform quantizers, to control the amount of discarded information; and iii) entropy coding, for lossless compression of quantized transform coefficients. The premise of this paper is that, while each of these modules is a highly effective compression tool in its own right, the full benefits will only be recouped if they are jointly optimized within a framework for $\mathrm{PQ}$ signal compression. We note, for example, that the Daubechies wavelet family is employed in most prior approaches, but it is unclear whether this choice represents a good match to PQ signals. Furthermore, the quantization approach is ad hoc with no justification for how the threshold for discarding coefficients (quantizing to zero) was selected.

Clearly, the true potential of these tools and the overall framework can only be achieved by joint optimization of the modules including, in particular, the wavelet family, quantizer intervals, and probability tables for entropy coding, for the actual statistics of the data.

In this paper, given the complexity of the cost function that controls the tradeoff between the conflicting objectives of rate (or compression ratio) and distortion, and optimizes numerous parameters, we employ a generic but powerful multi-objective genetic algorithm based optimization approach called the Non-dominated Sorting Genetic Algorithm (NSGA-II) [14]. The optimization is done separately for 8 classes of commonly occurring PQ disturbance signals, namely, flicker, harmonic distortion, impulse transient, notching, oscillatory transient, voltage interruption, voltage sag and voltage swell. Prior to compression, the signals are segmented as per [15] and 
classified as per [16], to learn parameters that are adaptive to the statistics of each type of disturbance. Wavelets from the families of Haar, Daubechies, Symlets, Coiflets and Biorthogonal are considered. Given the wavelets, the coefficients after decomposition are modeled by the Laplacian distribution, which has been observed in experiments to be an effective model (Laplacians are also commonly used in multimedia signal compression [17] [18]. Parameters are estimated for the "dead-zone plus uniform threshold" quantizer with nearly uniform reconstruction, which is optimal for the Laplacian distribution [19]. The quantizer parameters include the dead-zone interval width, width of the uniform intervals, and reconstruction offset within uniform intervals. Given the quantizer, the actual quantizer index statistics are used to learn the probability tables for entropy coding.

An initial population of 100 individuals are formed for the NSGA-II by randomly sampling the entire parameter space. The NSGA-II traverses the parameter search space based on individuals' fitness (or cost function) with respect to the objectives of compression ratio and distortion simultaneously. After certain number of generations, the NSGA-II converges to the most adapted wavelets for decomposition and the most appropriate parameters for quantization and entropy coding according to their compression ratio and distortion.

This paper is organized as follows: Section 2 covers the background of currently employed compression framework for PQ disturbance signals. Section 3 details our proposed methodology to jointly design all the parameters of the compression framework; Section 4 describes results obtained by our proposed approach; Finally, Section 5 presents conclusions of the presented research.

\section{PQ Signal Compression Framework}

The commonly employed basic framework for electric signals compression, depicted in Figure 1, includes three stages: i) transformation of the input signal to decorrelate coefficients in the transform domain; ii) quantization of the coefficients; and iii) entropy coding [7] [10] [11] [12] [13].

A WT is usually employed to generate the time-frequency representation coefficients. This transformation is performed by decomposing the signal into low and high frequency components, followed by temporal decimation by 2 . This decomposition is repeated recursively until the desired frequency and time resolution is achieved. Note that in each stage of decomposition, while the time resolution is decreased, the frequency resolution is correspondingly increased. This allows separating the frequency components introduced by a particular disturbance from those that are not related. These unrelated components can be then discarded by quantizing to zero without loss of quality during signal reconstruction, resulting in better compression [13]. Finally, all the quantized coefficients are losslessly entropy coded.

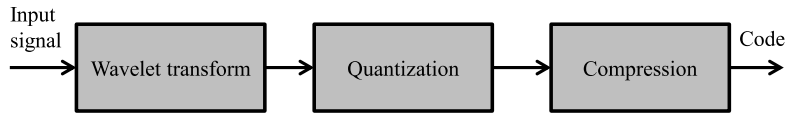

Figure 1: Current PQ signal compression framework.

\section{Joint Design of Updated PQ Signal Compression Modules}

As explained in Section 1, while the modules of the standard PQ compression framework can be very effective, their design choices were either arbitrary or unjustified. We thus propose updating the modules to account for variation in statistics of the data, and then propose a joint design approach of these modules.

\section{A. Adaptive Modules}

One key aspect that had largely been neglected by prior approaches is that statistics of different types of PQ disturbances can vary significantly. For example, the harmonic disturbance has components only at multiples of the fundamental frequency, but a transient disturbance has components spread across many more frequencies. Thus, in a significant departure from prior approaches, we propose employing modules that are designed specifically for each class of disturbance based on its statistics. In doing this, we are exploiting the fact that analysis of PQ signals requires segmentation and classification based on disturbances. This novel framework with classification preceding compression is practically feasible with availability of online segmentation [15] and classification [16] techniques that are tailored to these signals.

\section{B. Entropy Constrained Scalar Quantizers}

Another major shortcoming of current approaches is the ad hoc approach for quantization, where frequency coefficients are zeroed out based on an arbitrarily chosen threshold. Instead we propose to employ the right entropy constrained scalar quantizers (ECSQ), based on the empirically collected distributions of data for different disturbances. Figure 2 shows these distributions whose shapes are reasonably well modeled by the Laplacian distribution. Hence, we employ the dead-zone plus uniform threshold quantizer with nearly uniform reconstruction, which is the optimal ECSQ for Laplacian distributed data [9]. This quantizer is illustrated in Figure 4, and its structure is characterized by the dead zone width, width of uniform intervals, and the reconstruction offset in the uniform intervals. While it can be argued that the "zeroing out threshold" currently employed can capture the dead zone width, optimality will only be achieved once it is designed in conjunction with width of other intervals and offset for reconstruction in them. The other interesting observation in Figure 2 is the variation in statistics across disturbances, validating the proposed adaptation in Section A. 

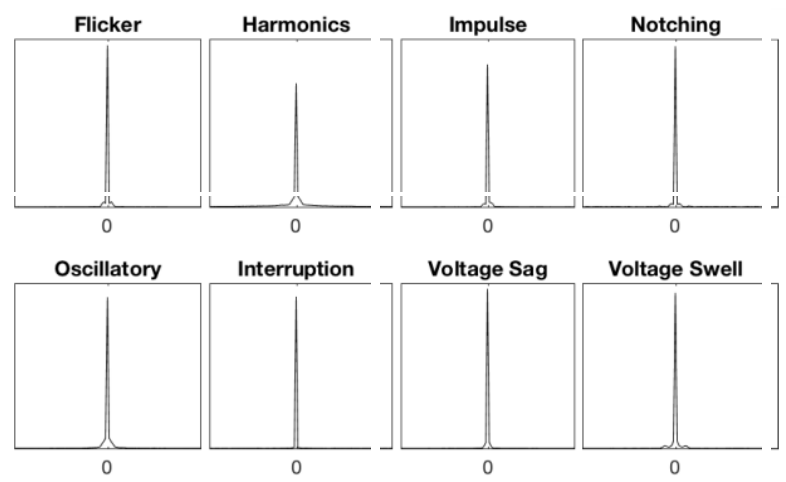

Figure 2: Empirical distribution for different disturbances.

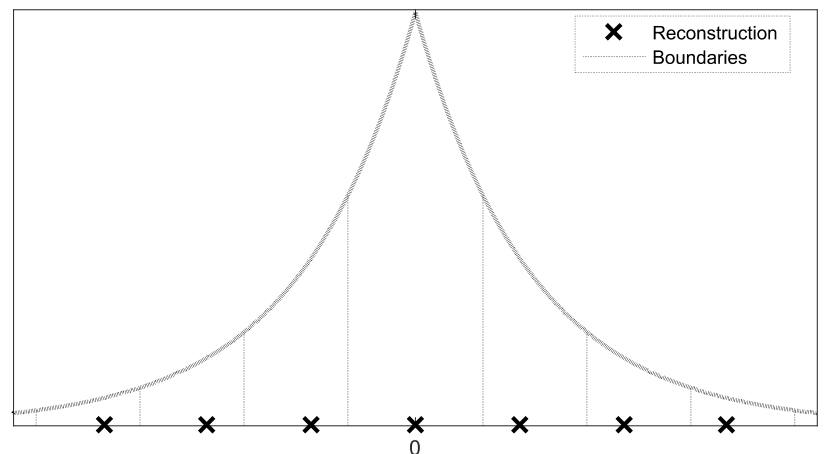

Figure 4: Dead-zone plus uniform threshold quantizer for Laplacian distribution.

\section{Joint Design of Modules Using Genetic Algorithm}

The final hurdle to realize the true potential of these tools and the overall framework is to jointly optimize all parameters of the system, including the wavelet family, Laplacian quantizer parameters, and probability tables for entropy coding, to match the actual data statistics. The objective is to find an approximation $\hat{\boldsymbol{f}}$ of the PQ signal of interest $\boldsymbol{f}$, encoded with $b$ bits, that minimizes the distortion $d(\boldsymbol{f}, \hat{\boldsymbol{f}})$. In this paper we employ the normalized mean squared error (NMSE) distortion metric as defined in [20].

Given the complexity of the cost function with two conflicting objectives of rate (or compression ratio) and distortion, and the variety of parameters, we employ a generic multi-objective genetic algorithm based optimization approach called NSGA-II [14]. Figure 3(a) and Figure 3(b) show all stages of the NSGA-II algorithm to determine the Pareto front [14], which represents the convex hull of the operational rate-distortion (RD) curve, for each one of the disturbance categories.

During the "Initialize population" stage of the algorithm, $N$ individuals are randomly selected from the entire parameter space. In "Fitness functions evaluation" stage, for each individual, all the training set samples go through the full compression and decompression steps using the parameters associated with that individual to determine the fitness, i.e., the cost functions of compression ratio and distortion, as presented in Figure 3(b). The first step is wavelet decomposition of PQ signals. This is followed by quantization of frequency coefficients. Given the quantized

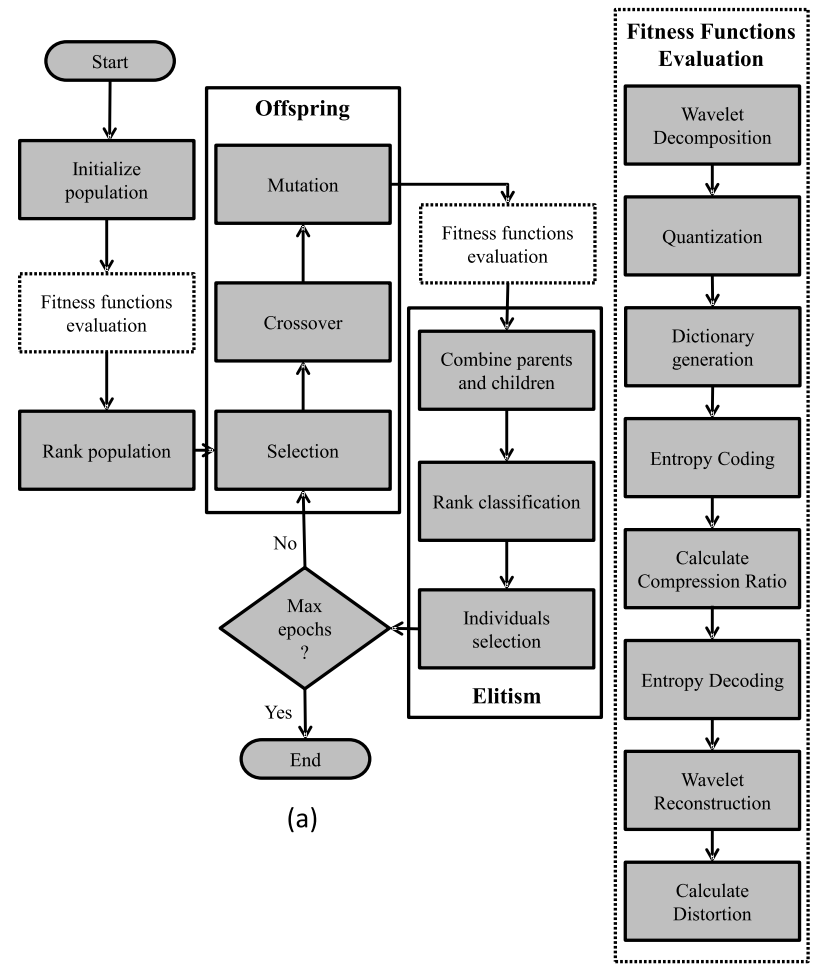

(b)

Figure 3: (a) -- NSGA-II overall procedure. (b) -- Detailed view of the Fitness Function Evaluation step.

coefficients, an entropy coding dictionary is generated using the actual probability distribution. This dictionary is used in entropy coding, which completes the encoding process to generate the bitstream. Given the bitstream, we calculate the compression ratio. The bitstream is then decoded by reversing the encoding steps to generate reconstructed PQ signals, given which we calculate the distortion.

In "Ranking population" stage, the NSGA-II employs nondominated sorting. Domination is defined as all objectives being not worse than that of the competing individual and at least one objective being strictly better.

Given this all individuals that are not dominated by others are ranked as front 1 . For our problem, this is picking individuals on the RD plane that lie on the bottom-left edge and connect the bottom most and left most point. All individuals only dominated by those from front 1 are ranked front 2, and so on [21]. In the "Selection" stage, parents who will be used to generate new offspring are chosen using tournament between two randomly selected individuals from the current population. The tournament winner is based on the front number, and if from same front, crowding distance, as defined in [21], is used to choose the individual from sparsely populated part of the front.

In every iteration, $N$ existing individuals (parents) generate $N$ new individuals (offspring) through simulated binary crossover and polynomial mutation [22]. Fitness function is then evaluated for all the offspring and the combined population of parents and children are ranked through nondominated sorting as before. Finally, the selection of $N$ individuals for the next generation/iteration is done through the tournament described before in the combined $2 \mathrm{~N}$ 
population. The algorithm is terminated after a fixed number of generations to obtain a Pareto front with $N$ best solutions, each with a wavelet family, quantizer and probability table for entropy coding. Note that during selection we maintain an external population of $25 \%$ (as suggested by [14]) to have adequate selection pressure for the elite solutions.

\section{Results}

The modules of the PQ signal compression are first designed using a training set, and then these modules are used with a test set to obtain the experimental results.

Mathematical models, which are well known to closely represent real world disturbances (as given in [23]), are used to generate 1,600 signals for each of flicker, harmonic distortion, impulse transient, notching, oscillatory transient, voltage interruption, voltage sag and voltage swell. Of the 1600 signals for each disturbance, half are used for training and remaining for testing. Synthetic data is used to ensure each class of disturbance has a broad coverage of variability in training data that no existing real dataset provided, while allowing a fair and easily reproducible comparison between competing approaches. Note that the proposed approach is generic and can be easily extended to other classes of disturbances.

The generated signals have a total duration of 10 cycles at $60 \mathrm{~Hz}$ fundamental frequency, and sampled at 128 points per cycle (i.e., $7.68 \mathrm{kHz}$, in line with prior research [24]). Note that segmentation [15] results in varying number of samples for each test signal.

The magnitude limits imposed (based on [25]) are -1 and 1 pu in steady-state, 0.1 and 0.9 pu for voltage sag, 1.1 and $1.8 \mathrm{pu}$ for voltage swell, 2 and 9 pu for transients, and for harmonic disturbance, up to 25 harmonics are allowed each between 0.07 and $0.2 \mathrm{pu}$. We evaluated both the Huffman coding and Arithmetic coding as entropy coding options. We used $N=100$ number of individuals in the genetic algorithm, which at the end of optimizations provides parameters for 100 operating points on the RD curve.
In the first experiment, we calculated the compression ratio achieved for each class of disturbance averaged over all test signals at a distortion of NMSE $=1.0 \times 10^{-5}$, which is the generally accepted standard in prior work [10] [11] [12] [13]. Results comparing the prior art, which uses nonadaptive basic threshold quantizer and Daubechies 4 wavelet, with the proposed approach of using jointly optimized adaptive ECSQ and wavelet transforms, is presented in Table 1, for both Huffman and Arithmetic coding options. We can clearly see that the proposed approach provides considerable improvement in compression ratio for all disturbances. While the average improvement over all disturbances is around $1.45 \mathrm{x}$, for the "Impulse transient" disturbance, the compression ratio is almost doubled. Amongst the entropy coders, the Arithmetic coder is the clear winner with an average compression ratio improvement of around $1.15 \mathrm{x}$.

To demonstrate the performance improvement specifically due to change in wavelet transform, we compared the performance of using the Daubechies 4 wavelets of prior art, with the Biorthogonal 3.1 wavelets, which was the best solution for all disturbances in our optimization approach, for both the Huffman and Arithmetic coding options.

The Pareto front (or the RD curve) averaged over all 8 disturbances, for the 4 variations is shown in Figure 5. Clearly, the optimally derived Biorthogonal 3.1 wavelet transform outperforms the previously used transform, providing around 10 to $15 \%$ reduction in rate (see blackdiamond versus red-circle curves, and green-triangle versus blue-square curves). Arithmetic coding provides further $15 \%$ reduction in rate when compared to Huffman coding (see black-diamond versus green-triangle curves, and redcircle versus blue-square curves). Overall, using Biorthogonal 3.1 wavelet transform with Arithmetic coding provides around $25 \%$ reduction in rate when compared to using Daubechies 4 wavelet transform with Huffman coding.

These results clearly demonstrate the effectiveness of the proposed updates to the PQ signal compression framework and the joint optimization approach.

Table 1 - Compression ratio comparison.

\begin{tabular}{|c|c|c|c|c|}
\hline \multirow[b]{4}{*}{ Disturbance } & \multirow{2}{*}{\multicolumn{4}{|c|}{$\begin{array}{r}\text { Distortion }(\mathrm{NMSE})=1.0 \times 10^{-5} \\
\quad \text { Compression }\end{array}$}} \\
\hline & & & & \\
\hline & \multicolumn{2}{|c|}{ Huffman coding } & \multicolumn{2}{|c|}{ Arithmetic coding } \\
\hline & Prior Art & Proposed Approach & Prior Art & Proposed Approach \\
\hline Flicker & $16: 1$ & $26: 1$ & $17: 1$ & $27: 1$ \\
\hline Harmonic distortion & $13: 1$ & $15: 1$ & $13: 1$ & $16: 1$ \\
\hline Impulse transient & $10: 1$ & $17: 1$ & $11: 1$ & $18: 1$ \\
\hline Notching & $12: 1$ & $17: 1$ & $13: 1$ & $17: 1$ \\
\hline Oscillatory transient & $14: 1$ & $20: 1$ & $15: 1$ & $22: 1$ \\
\hline Voltage interruption & $23: 3$ & $35: 1$ & $30: 1$ & $48: 1$ \\
\hline Volstage sage & $21: 1$ & $29: 1$ & $24: 1$ & $37: 1$ \\
\hline Voltage swell & 19:1 & $23: 1$ & $21: 1$ & $29: 1$ \\
\hline
\end{tabular}




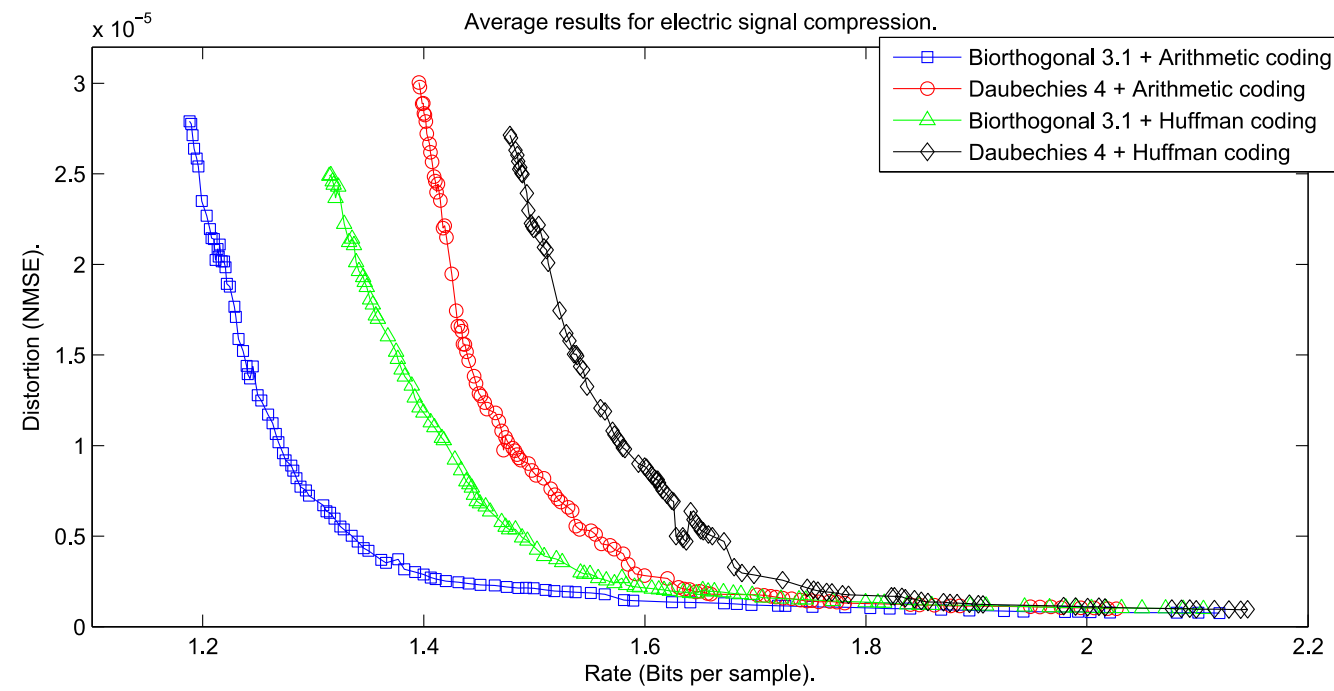

Figure 5: Performance comparison.

\section{Conclusion}

This paper demonstrated that jointly designing the PQ signal compression modules of wavelet transforms, nonuniform quantizers and entropy coding, using a genetic algorithm based optimization framework, resulted in significant improvement in PQ signals compression. Contrary to current approaches, which deploy "off the shelf" compression modules that were designed for other applications, the proposed approach leverages the ability to automatically classify the disturbances and designs the modules jointly while accounting for actual data statistics adapted to each category of disturbance. As a result of this joint optimization, Biorthogonal 3.1 emerged as the better choice of wavelet transform, in contrast with the currently employed Daubechies 4 wavelets. The overall experimental results demonstrate the significant performance improvement obtained by the proposed approach. Future research directions include, extending the adaptivity to hyper-local statistics, e.g., quantizers and entropy coders adapted to individual frequency components, and designing the modules for more categories of disturbances and their combinations.

\section{Acknowledgments}

The authors thankfully acknowledge the Electrical and Computer Engineering Departments of the São Carlos School of Engineering, University of São Paulo (Brazil) and of the University of California, Santa Barbara (USA), for the research facilities provided to conduct this project. The authors also acknowledge the financial support received from CAPES (Governmental Brazilian Institution).

\section{References}

[1] M. H. J. Bollen, I. Y. H. Gu, S. Santoso, M. F. Mcgranaghan, P. A. Crossley, M. V. Ribeiro and P. F. Ribeiro, "Bridging the gap between signal and power," IEEE Signal Processing Magazine, pp. 1231, 2009.

[2] L. Liboni, R. Flauzino, I. da Silva, E. Costa and M. Suetake, "Efficient signal processing technique for information extraction and its applications in power systems," Electric Power Systems Research, vol. 141, pp. 538-548, 2016.

[3] A. Vaccaro, I. Pisica, L. L. Lai and A. F. Zobaa, "A review of enabling methodologies for information processing in smart grids," International Journal of Electrical Power \& Energy Systems, vol. 107, pp. 516-522, 2019.

[4] F. Samie, L. Bauer and J. Henkel, "Edge computing for smart grid: An overview on architectures and solutions," Power Systems, pp. 21-42, 2019.

[5] P. F. Ribeiro, C. A. Duque, P. M. Ribeiro and A. S. Cerqueira, Power systems signal processing for smart grids, New York: John Wiley \& Sons, 2013.

[6] M. P. Tcheou, L. Lovisolo, E. A. B. d. Silva, M. A. M. Rodrigues and P. S. R. Diniz, "Optimum RateDistortion Dictionary Selection for Compression of Atomic Decompositions of Electric Disturbance Signals," IEEE Signal Processing Letters, vol. 14, no. 2, pp. 81-84, 2007.

[7] M. P. Tcheou, L. Lovisolo, M. V. Ribeiro, E. A. B. d. Silva, M. A. M. Rodrigues, J. M. T. Romano and P. S. R. Diniz, "The Compression of Electric Signal Waveforms for Smart Grids: State of the Art and Future Trends," IEEE Transactions on Smart Grid, vol. 5, no. 1, pp. 291-302, 2014.

[8] A. S. Spanias, "Speech coding: A tutorial review," Proceedings of the IEEE, vol. 82, no. 10, pp. 15411582, 1994.

[9] G. J. Sullivan, "Efficient scalar quantization of exponential and Laplacian random variables," IEEE Transactions on Information Theory, vol. 42, no. 5, pp. 1365--1374, 1996.

[10] J. Chung, E. J. Powers, W. M. Grady and S. C. Bhatt, "Variable rate power disturbance signal compression using embedded zerotree wavelet 
transform coding," in IEEE Power Engineering Society Winter Meeting, New York, NY, USA, 1999.

[11] C. T. Hsieh and S. J. Huang, "Disturbance data compression of a power system using the Huffman coding approach with wavelet transform enhancement," IEE Proceedings - Generation, Transmission and Distribution, vol. 150, no. 1, pp. 7-14, 2003.

[12] S.-J. Huang and M.-J. Jou, "Application of arithmetic coding for electric power disturbance data compression with wavelet packet enhancement," IEEE Transactions on Power Systems, vol. 19, no. 3, pp. 1334-1341, 2004.

[13] F. Lorio and F. Magnago, "Analysis of data compression methods for power quality events," IEEE Power Engineering Society General Meeting, vol. 1, pp. 504-509, 2004.

[14] K. Deb, A. Pratap, S. Agarwal and T. Meyarivan, "A fast and elitist multiobjective genetic algorithm: NSGA-II," IEEE Transactions on Evolutionary Computation, vol. 6, no. 2, pp. 182-197, 2002.

[15] L. C. M. Andrade, M. Oleskovicz and R. A. S. Fernandes, "Adaptive threshold based on wavelet transform applied to the segmentation of single and combined power quality disturbances," Applied Soft Computing, vol. 38, pp. 967 - 977, 2016.

[16] M. Oleskovicz, D. V. Coury, O. D. Felho, W. F. Usida, A. A. F. M. Carneiro and L. R. S. Pires, "Power quality analysis applying a hybrid methodology with wavelet transforms and neural networks," International Journal of Electrical Power \& Energy Systems, vol. 31, no. 5, pp. 206 212, 2009.

[17] M. Paez and T. Glisson, "Minimum mean-squarederror quantization in speech PCM and DPCM systems," IEEE Transactions on Communications, vol. 20, no. 2, pp. 225-230, 1972.

[18] R. Reininger and J. Gibson, "Distributions of the two-dimensional DCT coefficients for images,"
IEEE Transactions on Communications, vol. 31, no. 6, pp. 835-839, 1983.

[19] G. J. Sullivan, J. Ohm, W.-J. Han and T. Wiegand, "Overview of the high efficiency video coding (HEVC) standard," IEEE Transactions on Circuits and Systems for Video Technology, vol. 22, no. 12, pp. 1649-1668, 2012.

[20] M. F. Duarte, G. Shen, A. Ortega and R. G. Baraniuk, "Signal compression in wireless sensor networks," Philosophical Transactions of the Royal Society A: Mathematical, Physical and Engineering Sciences, vol. 370, no. 1958, pp. 118-135, 2012.

[21] P. Murugan, S. Kannan and S. Baskar, "Application of NSGA-II Algorithm to Single-Objective Transmission Constrained Generation Expansion Planning," IEEE Transactions on Power Systems, vol. 24, no. 4, pp. 1790-1797, 2009.

[22] R. B. Agrawal and K. Deb, "Simulated Binary Crossover for Continuous Search Space," Indian Institute of Technology, Kampur, India, 1994.

[23] R. Hooshmand and A. Enshaee, "Detection and classification of single and combined power quality disturbances using fuzzy systems oriented by particle swarm optimization algorithm," Electric Power Systems Research, vol. 80, no. 12, pp. 15521561, 2010.

[24] L. C. M. Andrade, M. Oleskovicz and R. A. S. Fernandes, "Analysis of Wavelet Transform applied to the segmentation of disturbance signals with different sampling rates," in IEEE Power Engineering Society General Meeting, National Harbor, MD, USA, 2014.

[25] R. C. Dugan, M. F. McGranaghan, S. Santoso and H. W. Beaty, Electrical Power Systems Quality, Third Edition, New York, NY, USA: McGraw-Hill Education, 2012. 